\title{
ADVERSE REACTIONS OF HYPERBARIC OXYGEN THERAPY - CASE DESCRIPTION OF A GENERALISED SEIZURE
}

\author{
Kinga Grobelska), ${ }^{1)}$ Monika Betyna ${ }^{2)}$, Ewa Zieliński $^{3)}$ \\ 1) Department of Emergency Medicine and Disaster Collegium Medicum in Bydgoszcz, Nicolaus Copernicus University in Toruń, Poland \\ 2) Department of Journalism, New Media and Social Communication at the University of Casimir the Great in Bydgoszcz, Poland \\ ${ }^{3)}$ Katedra Zdrowia Publicznego, Collegium Medicum im. L. Rydygiera w Bydgoszczy, Uniwersytet Mikołaja Kopernika w Toruniu
}

\section{ABSTRACT}

Hyperbaric oxygen therapy (HBOT) is a safe treatment, provided fulfilling certain rules of patient qualifications to treatment, as well as supervision over the course of therapy by qualified medical staff. Side effects reported in the literature are rare, and are usually mild and transient. Professional medical staff allows minimising the adverse events occurrence. The scale of complications is unknown, especially in Polish hyperbaric center. Careful analysis could be used to develop prevention procedures for patients of hyperbaric oxygen therapy. Hyperbaric Oxygen Centre and Wound Treatment in Bydgoszcz during 28 months performed hyperbaric oxygen therapy (HBOT) in case of 423 patients. During this period, adverse events occurred occasionally. 17 cases have been reported. Authors described study case 77 year-old patient who was admitted due to non- healing wound- left lower abdomen (state after radiotherapy). During the fourth session, on decompression phase patient have had a generalized seizure (tonic-clonic). The decompression was stopped, the oxygen supply was disconnected but only after the drug administration seizures terminated. The most likely causative agent of the adverse reactions of the patient treated with HBOT was the oxygen toxic effect on the brain tissue. However, analyzing the circumstances of the seizure termination: phase of decompression at the pressure 2ATA and lack of oxygen disconnection response, it cannot be excluded other causes of this complication.

Key words: Hyperbaric oxygen therapy, complications, seizure, Central Nervous System.

\section{ARTICLE INFO}

PolHypRes 2016 Vol. 54 Issue 1 pp. 19-22

ISSN: $1734-7009$ elSSN: 2084-0535

DOI: $10.1515 /$ phr-2016-0002

Pages: 4, figures: 0 , tables: 0

page www of the periodical: www.phr.net.pl

Publisher

Polish Hyperbaric Medicine and Technology Society

\section{Casuistic article (case study)}

Delivery date: $12.12 .2015 \mathrm{r}$

Date of approval for print: 12.01.2016r. 


\section{INTRODUCTION}

Hiperoxygental state brings about beneficial effects not only because of the delivery of oxygen to tissues and organs, but also because of the activation of biomolecules dependent production of free radicals. There is no doubt about the fact that HBOT is the initiator of the phenomenon of free radicals.

There is a vivid discussion about so-called "oxygen shock" during the hyperbaric oxygen therapy. It should be noted, however, that the phenomenon is accompanied by activation of antioxidant hence authors refer to this phenomenon as a controlled oxygen shock. The effect of HBOT, resulting from the increased amount of hiperbaric oxygen reaching the tissues and organs, may also be the cause of less common side toxic effects on the lungs and central nervous system.

Symptoms of pulmonary toxicity as a cough, retrosternal pain, breathing difficulties or shortness of breath are rare and the nature of the symptoms allows to completion the exposure before the appearance of the patient's health-threatening complications $[1,2,3]$. In the case of brain tissue oxygen toxicity the side effects are manifested, nearly always, as a seizure attack. The Hyperbaric Oxygen Therapy and Wound Treatment Centre reported one such case that has been described below.

\section{Case STUdY}

A 77 year-old patient admitted due to nonhealing wound- left lower abdomen from about 3 months. In May 2015 surgical procedure to remove sarcoma recurrence around the left lower abdomen with muscle and pubic bone periosteum as well as reconstruction of hernias using mesh. The postoperative course was uncomplicated. Microscopic examination: spindle-cell sarcoma with areas of necrosis and chronic inflammatory infiltration (state after radiotherapy).

The patient was admitted to the Hyperbaric Oxygen Therapy and Wound Treatment Centre on $08 / 03 / 2015$. Thirty sessions of hyperbaric oxygen therapy (90min / 2.5 ATA / 100\% oxygen) were scheduled. On admission, the left groin wound length was of $130 \mathrm{~mm}$ and a maximum width of $60 \mathrm{~mm}$ and a depth of $50 \mathrm{~mm}$. Within the wound fibrin, necrosis and signs of inflammation occurred. A significant amount of purulentserous exudate from the wound was reported. From about a year continuing ailments around the wound are observed- 5 points (NRS scale), periodically very severe (NRS 8-9). The patient took Ketoprofen and Tramadol chronically. The microbiological examination of wound swabs (collected on 08/04/2015), Klebsiella pneumoniae, sensitive to all antibiotics.

The first session of hyperbaric oxygen therapy proceeded without any complications; the patient had no problems with equalizing pressure in the middle ear. Also he did not report any discomfort both during and after the session. In the next two days, the treatment was proceeded without any side effects. During the fourth session, on decompression phase patient have had a generalized seizure with stiffness all over the body, loss of consciousness and urination.

The attack of seizure was not complicated by the fall or injury. After stopping the chamber work, the patient was placed on the floor, intravascular access (external jugular vein) was performed and $10 \mathrm{mg}$ of diazepam was given. After about 5 minutes the convulsions subsided. After the decompressed chamber, patient was carrying and lying on a trolley and then started monitoring the vital functions (ASM 80 / min, RR 110/60; Sp02 97\%), glucose $120 \mathrm{mg} \%$. Neurological examination occurred without any paralysis. A gradual clearing of consciousness was observed. The patient was transferred to the emergency department in order of diagnosis and further possible treatment. Due to CNS evaluation the patient was disqualified from HBOT treatment.

\section{DISCUSSION}

Mechanisms of hyperbaric oxygen therapy are based on phenomena related to the reduction of air bubbles dependent on pressure as well as on the effect of maximum tissues oxygenation. The oxygen toxic effect on the central nervous system manifests, most frequently, as seizures attack. The first time it was described by Paul Bert in 1878 and since then many studies concern the influence of hyperbaric oxygen therapy on the nervous system.

Hyperbaric oxygen treatment particularly predisposes to the enhancement of the oxygen level in brain tissue. It also causes, besides positive medicinal properties, some side effects. The risk of convulsions, in studies until 1990, has been set in 1 per 10,000 compressions. Plafki and colleagues as well as Hampson and Atik described the risk of seizures as a complication of CNS oxygen toxicity determined by the level 3 per 10 000 patients. In 2004 Yildiz and colleagues assessed the incidence of seizures in the largest group, more than 80,000 patients treated in a hyperbaric chamber at more than 2ATA pressure, breathing $100 \%$ oxygen.

They found the attack of seizures only in 2 cases. The causes of discrepancies in test results were unknown. It should be noted that the manifestation of CNS oxygen toxic effects in the convulsion form is a rare case.

In the Hyperbaric Oxygen Centre and Wound Treatment in Bydgoszcz there were 423 patients treated since 2013. Discomfort during compression was reported by 13 people. Only in one case the patient experienced hyperbaric oxygen therapy adverse reactions in the seizures form. The other 12 cases were laryngological complications: ear pain and bleeding from the nose.

The oxygen toxic effect on the CNS is highly dependent on the conditions of compression. The higher ambient pressure and longer oxygen therapy time, the earlier symptoms of brain tissue toxify. Welslau and Almeling in their studies compared two groups of patients where treatment was conducted for 60 and 90 minutes. The longer time of oxygen therapy, the higher rates of seizures were reported [4]. In the HbOT Centre patients are treated in accordance with hyperbaric oxygen therapy standard procedures. Patients in the 2.5 ATA pressure environment breathe oxygen for 60 minutes. In order to minimize oxygen toxic effects on lung tissue and brain after a 20 minutes period of breathing $100 \%$ oxygen occurs 5 minute air break.

During decompression period to 1,4 ATA pressure patient breathe oxygen through the mask. The sessions in the hyperbaric chamber are taking place once a day. In the presented case, these hyperbaric chamber conditions was also applied. During the decompression, at 
pressure 2 ATA, of the HBOT fourth session generalized seizure was observed. The Interruption of oxygen supply did not discontinue seizure activity, it was observed until the drug administration - Diazepam by the doctor accompanying the patient in a hyperbaric chamber.

The drug was given about 7 minutes from the attack beginning and six minutes from mask removed. There were not observed prodromal symptoms. In 2013 the patient was diagnosed with spermatic cord spindlecell sarcoma. During the qualification no metastasis or other factors increase the risk of seizures activity were not found, therefore should be assumed that the oxygen toxic effect on the brain tissue was the most likely causative factor. Analyzing the circumstances of the seizure, the decompression phase and the lack of the disconnection of oxygen response, other causes of this complication cannot be excluded.

Mechanisms of hyperbaric oxygen therapy are based on phenomena related to the reduction of air bubbles dependent on pressure as well as on the effect of maximum tissues oxygenation. The undesirable effects are an integral part of every medical therapy. During hyperbaric oxygen therapy a variety of complications also occurs. These are mostly rare or transient cases. Professional medical staff allows minimizing the adverse events occurrence. The scale of complications is unknown, especially in Polish hyperbaric center. Careful analysis could be used to develop prevention procedures for patients of hyperbaric oxygen therapy.

\section{BIBLIOGRAPHY}

1. Thorsen E1, Aanderud L, Aasen TB. Effects of a standard hyperbaric oxygen treatment protocol on pulmonary function. Eur Respir J. 1998 Dec;12(6): 1442-5;

2. Mialon P1, Barthélémy L, Michaud A, Lacour JM. Pulmonary function in men after repeated sessions of oxygen breathing at $0.25 \mathrm{MPa}$ for 90 min. Aviat Space Environ Med. 2001 Mar;72(3):215-8;

3. Pott F1, Westergaard P, Mortensen J, Jansen EC. Hyperbaric oxygen treatment and pulmonary function. Undersea Hyperb Med. 1999 Winter; 26(4):225-8;

4. Welslau W, Almeling M. Center for Diving and Hyperbaric Medicine, Am Roten Kreuz Krankenhaus, Kassel, Germany. Strahlentherapie und Onkologie: Organ der Deutschen Rontgengesellschaft .1996, 172 Suppl 2:10-12.

Kinga Grobelska Ph.D.

Department of Emergency Medicine and Disaster Collegium Medicum in Bydgoszcz,

Nicolaus Copernicus University in Toruń

tel 606270177

mail: kingro@poczta.onet.pl 